\title{
A case of pararenal angiomyolipoma arising from an existing exophytic angiomyolipoma
}

\author{
Michael Lipsky, BS; Ingride Richardson, MD; Bassir Tareen, MD
}

\begin{abstract}
A 52-year-old healthy woman with a congenital solitary right kidney presented with an incidentally found pararenal mass. Computed tomography and magnetic resonance imaging revealed an exophytic mass in the right kidney consistent with an angiomyolipoma and an additional enhancing pararenal mass that contained fatty elements. Differential diagnosis of the mass included liposarcoma and angiomyolipoma. Upon exploration, the exophytic angiomyolipoma was found to be contiguous with the pararenal mass via an isthmus of fatty tissue. Frozen sections were taken from both masses which confirmed that both were angiomyolipoma. This is the first case reported in the literature wherein an angiomyolipoma extended extrarenally and formed a significantly larger pararenal mass.
\end{abstract}

Can Urol Assoc J 2010;4(3):E79-E81

\section{Introduction}

Angiomyolipoma (AML) is a benign tumour composed of fat, muscle and blood vessels. These tumours generally present within the kidneys, but there are reports in the literature of extrarenal $\mathrm{AML}$, most commonly occurring in the liver and uterus. ${ }^{1}$ Few reports have described an extrarenal extension of an AML.

Two different types of AMLs have been described: fatpoor and classic. Fat-poor AML have been shown to evade radiographic diagnosis because of their lack of lipid component. ${ }^{2}$ While classic AML demonstrate all three components on histology, in certain cases one of the three constituents may dominate. Of note are the epithelial cell dominated AMLs, which are a more aggressive subtype that have the potential to metastasize. ${ }^{3}$ Some cases of AML can often be difficult to distinguish from liposarcoma, ${ }^{4}$ as both types of tumours have fatty components. We report a case of a suspected angiomyolipoma in which a suspicious extrarenal extension of the mass prompted further investigation.

\section{Case report}

A 52-year-old woman, with a history of microhematuria, was referred for evaluation of two suspicious renal masses on the right side. Past medical history was significant for a congenital solitary kidney. The patient had no other complaints and abdominal exam was benign. The results of laboratory studies including biochemistry and differential blood cell count were within normal limits. Abdominal computed tomography $(\mathrm{CT})$ scan revealed a right renal mass and a right pararenal mass (Fig. 1a) (Fig. 1b). Magnetic resonance imaging studies confirmed that the pararenal mass enhanced with administration of contrast and also contained fatty elements (Fig. 2). The small exophytic renal mass was consistent with AML (Fig. 1b). The larger pararenal mass was more concerning; based on radiologic imaging it was difficult to determine whether the mass was a liposarcoma, which would require excision, or an AML which could simply be observed. It could not be determined whether the pararenal mass was in any way related to the exophytic AML.

A CT-guided biopsy was considered but declined because of the significant risk of bleeding and also due to the potential for inconclusive diagnosis on fine needle aspiration. Also, due to the heightened concern of a solitary kidney, a retroperitoneal exploration with possible partial nephrectomy was performed. Intraoperatively, it was noted that the two aforementioned masses were continuous (Fig. 3). The larger pararenal portion, a well-circumscribed $9.8 \times 4.8 \times 2.4 \mathrm{~cm}$ yellow, soft mass, was excised from the right kidney. The histological finding was consistent with AML. Frozen sections of the smaller mass were also found to be benign. Partial nephrectomy was declined. The patient's recovery was uneventful.

\section{Discussion}

Angiomyolipomas are benign neoplasms composed of variable amounts of blood vessels, fat and muscle tissue. They make up $1 \%$ of all renal masses and most often appear in middle-aged women as was seen in this case. ${ }^{5}$ Classic AML may arise sporadically or can be associated with tuberous 


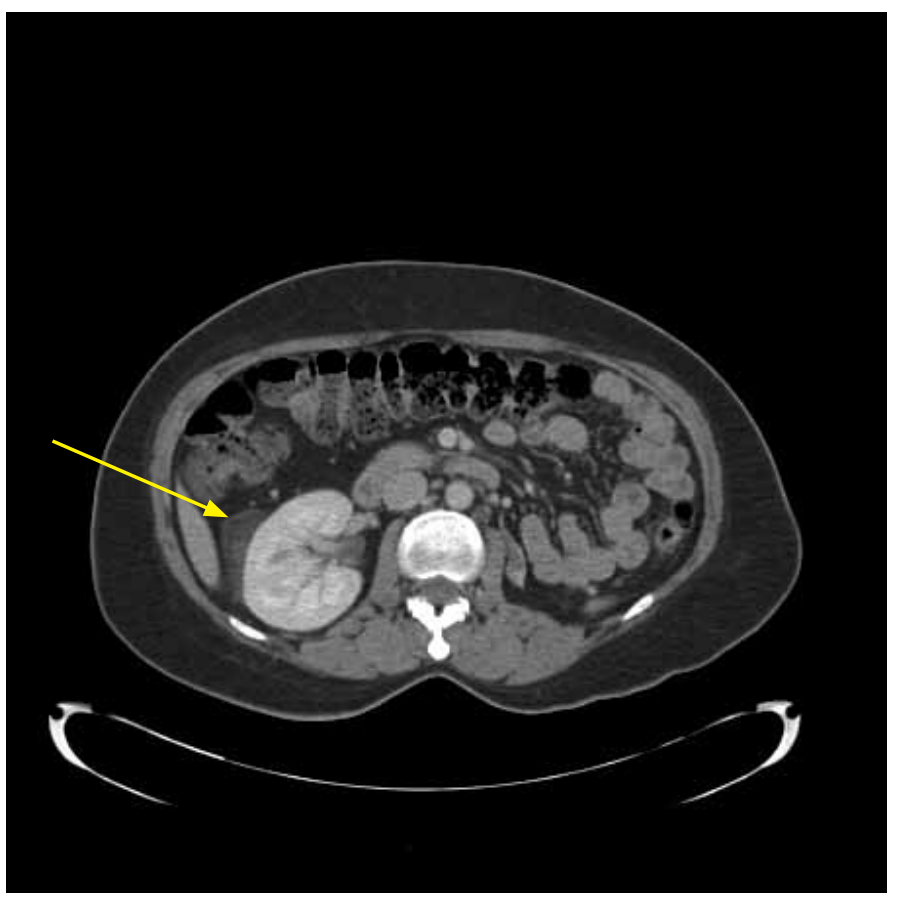

Fig. 1a. A computed tomography identification of the 2 masses (shown by the arrow).

sclerosis. With an overall incidence of $0.07 \%$ to $0.3 \%$ of the general population, it is estimated that over 10 million people worldwide have renal AML with one tenth of these people also having tuberous sclerosis. ${ }^{6-8}$ As AMLs enlarge, they become more vascular and more prone to hemorrhaging, which can be life-threatening. In addition, flank pain, hematuria and hypertension are common symptoms of AML. Diagnosis is often made radiologically with confirmation made upon histological review. When AML is found in the retroperitoneal space, it may be difficult to distinguish this benign tumour from malignant tumours such as liposarcoma and renal cell carcinoma. ${ }^{4}$ However, it is important to make the distinction between the two because liposarcoma is malignant and should be widely excised.

The differential diagnosis of lipid-containing masses also includes lipoma and leiomyoma with fatty change. Two reports describe useful methods to differentiate between AML and liposarcoma radiologically. ${ }^{9,10}$ Israel and colleagues describe three major imaging findings that can help in the diagnosis. ${ }^{9}$ They write that a defect in the renal parenchyma should be present at the origin of the AML, whereas a liposarcoma is more likely to displace and compress the kidney. Angiomyolipomas also usually contain enlarged vessels. Finally, the presence of other fatty lesions or another concomitant $\mathrm{AML}$ is a strong indicator of non-malignant potential of the mass in question.

Ellingson and colleagues also indicate that a parenchymal defect is associated with AML. ${ }^{10}$ In their small series, they noted that a tumoural vessel extended into the renal cortex

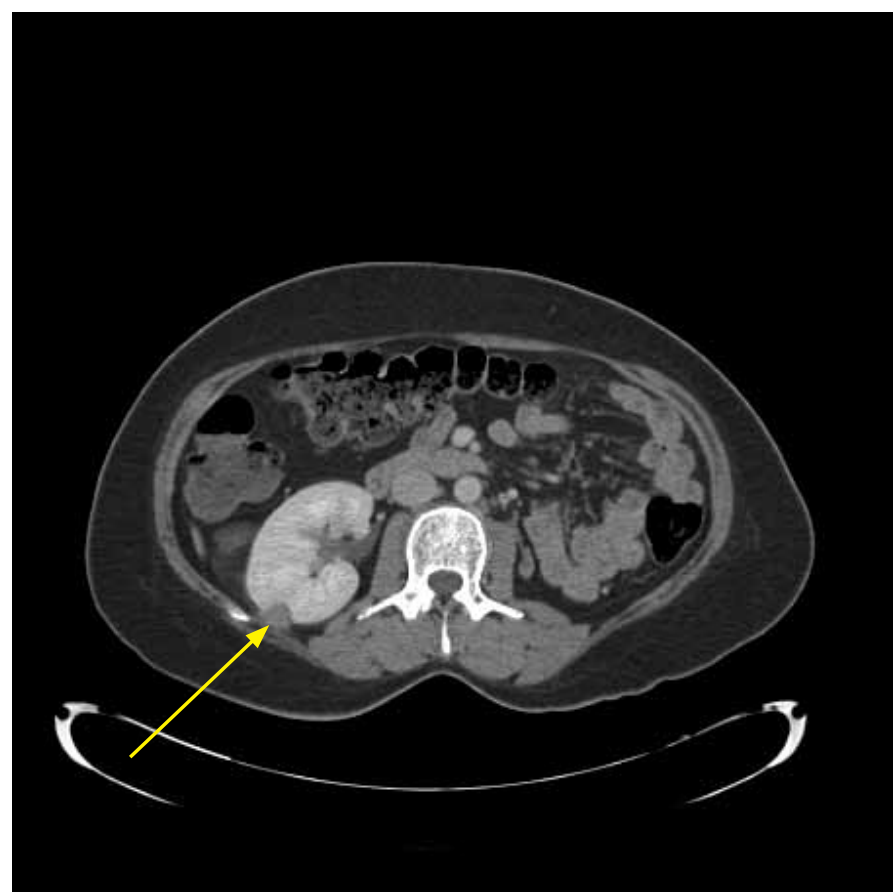

Fig. 1b. A computed tomography identification of the 2 masses (shown by the arrow).

in 7 of 9 AMLs but not in any liposarcomas, and that calcifications were seen in 6 of 11 liposarcomas but were not present in any AMLs. As demonstrated by this case, however, the distinction between liposarcoma and AML could not be made radiographically even with these suggestions. The mass did not contain any vessels or calcifications and, though there was no parenchymal defect, the mass was confirmed to be an AML.

In addition to radiographic differentiation between $\mathrm{AML}$ and other renal tumours, immunohistochemical staining after biopsy may be used to make a definitive diagnosis of AML. Ashfaq and colleagues performed an analysis of the immunostaining profile of 72 renal tumours including $A M L$, leiomyoma, leiomyosarcoma, liposarcoma and a number of other renal tumours. They conclude that of all the renal tumours tested, only AML contain reactivity to human melanosome-associated protein (HMB-45) and that this specific reactivity can be used to distinguish this tumour from other renal tumours. ${ }^{11}$

When treating angiomyolipoma, the ultimate goal is the preservation of renal function. Selective arterial embolization is the treatment of choice when the AML is large and likely to hemorrhage. ${ }^{12}$ Radiofrequency ablation and partial nephrectomy are two other nephron-sparing treatment modalities. ${ }^{13}$ Conservative "watch-and-wait" strategies with regular follow-up are preferred for asymptomatic patients with small masses or those in whom the aforementioned treatment options are not feasible. ${ }^{14}$ 


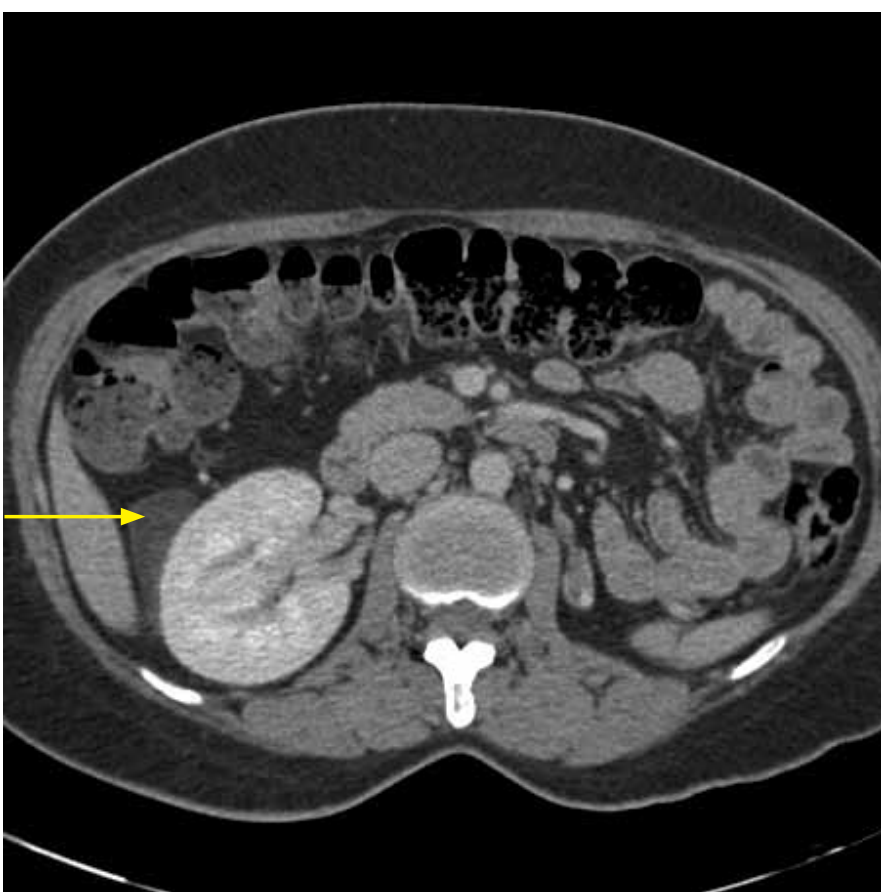

Fig. 2. A magnetic resonance imaging of the pararenal mass (shown by arrow).

\section{Conclusion}

Our description of a suspicious pararenal mass arising from an AML has not previously been reported in the literature to our knowledge. This case was further complicated by the circumstance of a solitary kidney. These conditions prompted surgical exploration with a favourable result for this patient. Greater developments and advances in renal imaging are still needed to better delineate benign from malignant pararenal masses.

Albert Einstein College of Medicine, Beth Israel Medical Center, Department of Urology, New York, NY

Competing interests: None declared.

This paper has been peer-reviewed.

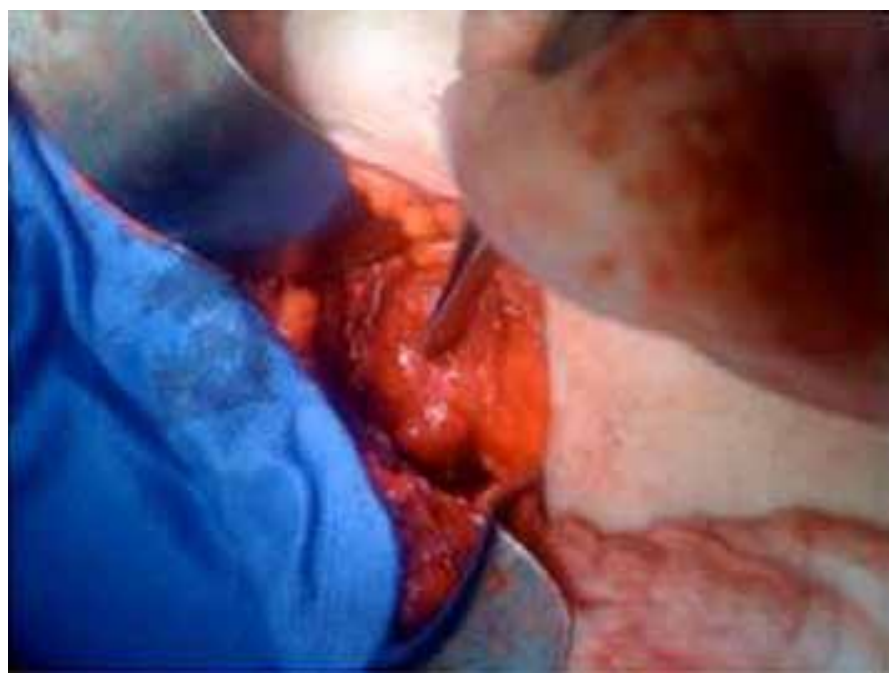

Fig. 3. The back end of the forceps demonstrates the intrarenal angiomyolipoma.

\section{References}

1. Ditonno P, Smith R, Koyle M, et al. Extrarenal angiomyolipomas of the perinephric space. J Urol 1992; 147:447-50

2. Lane BR, Aydin H, Danforth TL, et al. Clinical Correlates of Renal Angiomyolipoma Subtypes in 209 Patients: Classic, Fat Poor, Tuberous Sclerosis Associated and Epithelioid. J Urol 2008;180:836-43.

3. Gupta C, Malani AK, Gupta V, et al. Metastatic retroperitoneal epitheliod angiomyolipoma. J Clin Pathol 2007;60:428-31.

4. Frank I, Takahashi S, Tsukamoto T, et al. Genitourinary Sarcomas and carcinomas in adults. In: Vogelzang $\mathrm{N}$, ed. Comprehensive Textbook of Genitourinary Oncology. 2nd ed. Philadelphia: Lippincott Williams \& Wilkins; 2000:1102-19.

5. Tsutsumi $M$, Yamauchi A, Tsukamoto $S$, et al. A case of angiomyolipoma presenting as a huge retroperitoneal mass. Int J Urol 2001;8:470-1.

6. Hajdu S, Jr. FF. Angiomyolipoma of the kidney: report of 27 cases and review of literature. J. Urol 1969;102:396.

7. Bissler JJ, Kingswood JC. Renal Angiomyolipomata. Kidney Int 2004;66:924-34

8. Taylor R, Joseph D, Kohaut E, et al. Renal Angiomyolipoma associated with lymph node involvement and renal cell carcinoma in patients with tuberous sclerosis. J Urol 1989;141:930-2.

9. Israel GM, Bosniak MA, Slywotzky CM, et al. CT Differentiation of Large Exophytic Renal Angiomyolipomas and Perirenal Liposarcomas. AJR Am J Roentgenol 2002;179:769-73.

10. Ellingson JJ, Coakley FV, Joe BN, et al. Computed Tomographic Disctinction of Perirenal Liposarcoma from Exophytic Angiomyolipoma: A Feature Analysis Study. J Comput Assist Tomogr 2008;32:548-52.

11. Ashfaq R, Weinberg AG, Albores-Saavedra J. Renal Angiomyolipomas and HMB-45 Reactivity. Cancer 1993;71:3091-7.

12. Nelson C, Sanda M. Contemporary Diagnosis and Management of Renal Angiomyolipoma. J Urol 2002;168:1315-25.

13. Prevoo W, van den Bosch MA, Horenblas S. Radiofrequency Ablation for Treatment of Sporadic Angiomyolipoma. Urology 2008;72:188-91.

14. Sooriakumaran P, Gibbs P, Coughlin $G$, et al. Angiomyolipomata: challenges, solutions, and future prospects based on over 100 cases treated. BJU Int 2010;105:101-6.

Correspondence: Dr. Basir Tareen, Urologic Oncology, Beth Israel Medical Center, 10 Union Sq. East, Suite 3A, New York, NY 10003; btareen@chpnet.org 